\title{
Article \\ A Flexible Turning and Sensing System for Pressure Ulcers Prevention
}

\author{
Ying Zhang ${ }^{1,+}$, Xiaofeng Zou ${ }^{2,+}$, Bin Zhang ${ }^{2}$, Yi Han ${ }^{3}$, Shuoyu Wang ${ }^{3}$, Tao Liu ${ }^{2, *}$ and Xiufeng Zhang ${ }^{4, *}$ \\ 1 EN-SE (Jiaxing) Co., Ltd., Building 3, No.235 Chengbei Road, Jiading Dist., Shanghai 201800, China; \\ amy.zhang@en-se.com \\ 2 State Key Laboratory of Fluid Power and Mechatronic Systems, School of Mechanical Engineering, \\ Zhejiang University, Hangzhou 310027, China; xf_zou@zju.edu.cn (X.Z.); zjuzhangbin@zju.edu.cn (B.Z.) \\ 3 Department of Intelligent Mechanical Systems Engineering, Kochi University of Technology 185 \\ Miyanokuchi, Tosayamada-Cho, Kami 782-8502, Kochi, Japan; 258012g@gs.kochi-tech.ac.jp (Y.H.); \\ wang.shuoyu@kochi-tech.ac.jp (S.W.) \\ 4 Key Laboratory of Rehabilitation Technical Aids Technology and System of the Ministry of Civil Affairs, \\ National Research Center for Rehabilitation Technical Aids, Beijing 100000, China \\ * Correspondence: liutao@zju.edu.cn (T.L.); zhangxiufeng@hit.edu.cn (X.Z.); Tel.: +571-87951314-6221 (T.L.) \\ + The two authors contributed equally to this work and should be considered co-first authors.
}

check for updates

Citation: Zhang, Y.; Zou, X.; Zhang, B.; Han, Y.; Wang, S.; Liu, T.; Zhang, X. A Flexible Turning and Sensing System for Pressure Ulcers Prevention. Electronics 2021, 10, 2971. https://doi.org/10.3390/ electronics10232971

Academic Editor: Maciej Ławryńczuk

Received: 2 November 2021

Accepted: 26 November 2021

Published: 29 November 2021

Publisher's Note: MDPI stays neutral with regard to jurisdictional claims in published maps and institutional affiliations.

Copyright: (c) 2021 by the authors. Licensee MDPI, Basel, Switzerland. This article is an open access article distributed under the terms and conditions of the Creative Commons Attribution (CC BY) license (https:// creativecommons.org/licenses/by/ $4.0 /)$.

\begin{abstract}
Pressure ulcers (PU) are one of the most frequent hazards of long-term bedridden patients With the continuous increase of aging, the number of long-term bedridden disabled and semidisabled elderly people is increasing. At the same time, there is a serious shortage of professional pressure ulcer nursing staff. There is also a lack of flexible turning equipment for PU prevention. The research in the field of pressure ulcer prevention at home and abroad is carried out steadily, and the equipment for turning over by pneumatic or mechanical drive is developed. However, these devices often have insurmountable defects, such as complex structure, cost constraints, difficult control, weak body feeling, and so on. Under these circumstances, a set of pneumatic turnover mattresses based on clinical nursing methods have been developed. The mattress is divided into a turnover area and two support areas. The turnover airbag is linked with the support airbag to improve the patient's comfort when passively turning over. The turnover amplitude and interval can be adjusted to provide a personalized turnover experience for bedridden patients. To improve the safety of the turning mattress during automatic turning, we also add a temperature sensor based on the principle of infrared reflection to monitor the status of bedridden patients, which can realize real-time temperature measurement, monitoring of getting out of bed and monitoring of the turning process.
\end{abstract}

Keywords: pressure ulcer prevention; automatic repositioning; temperature monitoring; support airbag; flexible turning

\section{Introduction}

With the growth of the aging population, the number of disabled and semi-disabled elderly people is increasing [1]. Pressure ulcers (PU) are one of the most frequent hazards for patients who are bedridden for a long time [2,3]. These people need the intervention of nurses to assist in turning over to improve the pressure distribution and achieve the purpose of PU prevention [4,5]. However, many problems are present such as the shortage of caregivers, the high average age of caregivers, and the low professional quality of caregivers. It is difficult to meet the patient's turnover care needs.

It is generally believed that the combined effect of pressure, friction and shear force on the human body caused by elderly patients lying in bed is the main cause of PU. Urinary incontinence, excessive sweating, and hot and humid local environment are also important causes of PU. Therefore, clinically, patient repositioning is usually the main means to prevent PU in nursing settings. Turning over care for patients once every two hours is 
stressful not only for the nursing staff in nursing institutions and hospitals, but also for the nursing staff at home [6].

There is a serious shortage of nursing professionals all over the world. According to the Federation of Nurses and Health Care Professionals, there are more than 20,000 elderly care workers in China, but the demand is about 10 million, and the gap is huge. Coupled with the high rate of nursing staff, long shifts, back and shoulder injuries and other groups of poor health conditions, hospitals and elderly care institutions are facing increasing pressure in attracting and retaining nursing staff $[7,8]$. According to the survey results, the age of some nursing staff in some elderly care institutions is generally over 65 , and the level of cultural literacy is generally low. The elderly and infirm are unable to meet the mental and physical needs of nursing, and cannot perform scientific nursing work in daily nursing care. For elderly patients with PU, there are phenomena such as irregular operation, and there is a risk of secondary injury to vulnerable elderly patients [9].

The products on the market for pressure ulcer prevention are mainly anti-decubitus mattresses [10], which disperse the pressure of the patient when lying in bed by using static latex, and other material mattresses or dynamic pneumatic mattresses to achieve a certain pressure ulcer prevention [11,12]. Related research designed to use pressure sensors, temperature and humidity sensors to monitor the local state of the patient's body in bed, and then feedback control is performed by multiple independent execution units to improve the pressure distribution of the human body $[13,14]$. Among them, the "Taiatsu Bunsan" mattress developed by Japanese institutions is representative. The mattress is composed of 108 independent stacked airbags. The self-made rubber pressure sensor is used to perform real-time pressure detection on bedridden patients. The surface of the mattress will be based on the patient's body posture and pressure distribution automatically adjust the air pressure of the corresponding position of the airbags to maintain a relatively even distribution of body pressure [15].

In addition, Yousefi et al. [16] adopted a mechanical-pneumatic hybrid method to develop a new type of mattress for PU prevention, which can realize the function of turning on the basis of monitoring pressure. However, the above-mentioned smart mattresses often have insurmountable defects, such as complex structures and cost constraints, which are difficult to use widely. In daily nursing care, the turning-over method is the main manual intervention method for PU prevention. It must be matched with a suitable decompression support mattress to achieve a good pressure ulcer prevention effect.

According to the international "Pressure Ulcer Prevention and Treatment: Clinical Practice Guidelines", the key prevention strategies of pressure ulcer include risk assessment, the use of support surfaces, regular posture adjustment, skin care and nutritional care. Pressure ulcer prevention products at home and abroad mainly focus on the purpose of "sharing body pressure on support surface", including static flexible support material, pneumatic alternating pressure relief mattresses, etc. [17]. Some studies expect to replace or partially replace manual intervention by equipment for turning over operation, but the equipment often has insurmountable defects, which makes it difficult to apply them in a short time. Pressure ulcer is the most likely to occur on the back, elbows, ankles, heels, hips, buttocks, shoulders and head [4]. In daily care, two hours-per-time-turnover-operation is the main manual intervention method for pressure ulcer prevention, and it should be combined with a suitable decompression support mattress to achieve a good pressure ulcer prevention effect. Regular turning over is still the main method to prevent PU [18,19].

In recent years, with the development of technologies in computers and Internet of Things, sensor technology has been rationally applied in the field of PU prevention, and the equipment shows a trend of intelligent development [20]. In addition, research on the realtime monitoring of the pressure and various physiological parameters of bedridden patients is underway [21]. With the continuous deepening of the application of digitalization in medical care, PU prevention can be used as an independent functional module of the digital cloud platform [22]. Real-time monitoring of patient signs and intelligent equipment instead of nursing staff to perform nursing operations such as turning over, this is the 
future of pressure ulcer prevention of smart medical care [23-25]. Since the COVID-19 epidemic spread, temperature measurement is one of the effective means of epidemic prevention. Based on actual needs, we propose that the temperature sensor is applied to the turning mattress to monitor the condition of bedridden patients. As shown in Figure 1, we developed a flexible turning and sensing system for pressure ulcer prevention, which is mainly composed of turning mattresses and monitoring equipment. The mattress is driven by a flexible material to turn over, and a reasonable turning method is designed to prevent pressure sores and increase the safety and comfort of the process. We applied the non-contact temperature sensor based on infrared reflection principle to the mattress, and measured the bed temperature of the elderly by measuring the forehead temperature. For this reason, we designed a calibration method to improve the accuracy of the sensor. Through real-time temperature monitoring, body temperature detection, monitoring of getting out of bed, and status monitoring of the process of turning over are realized.

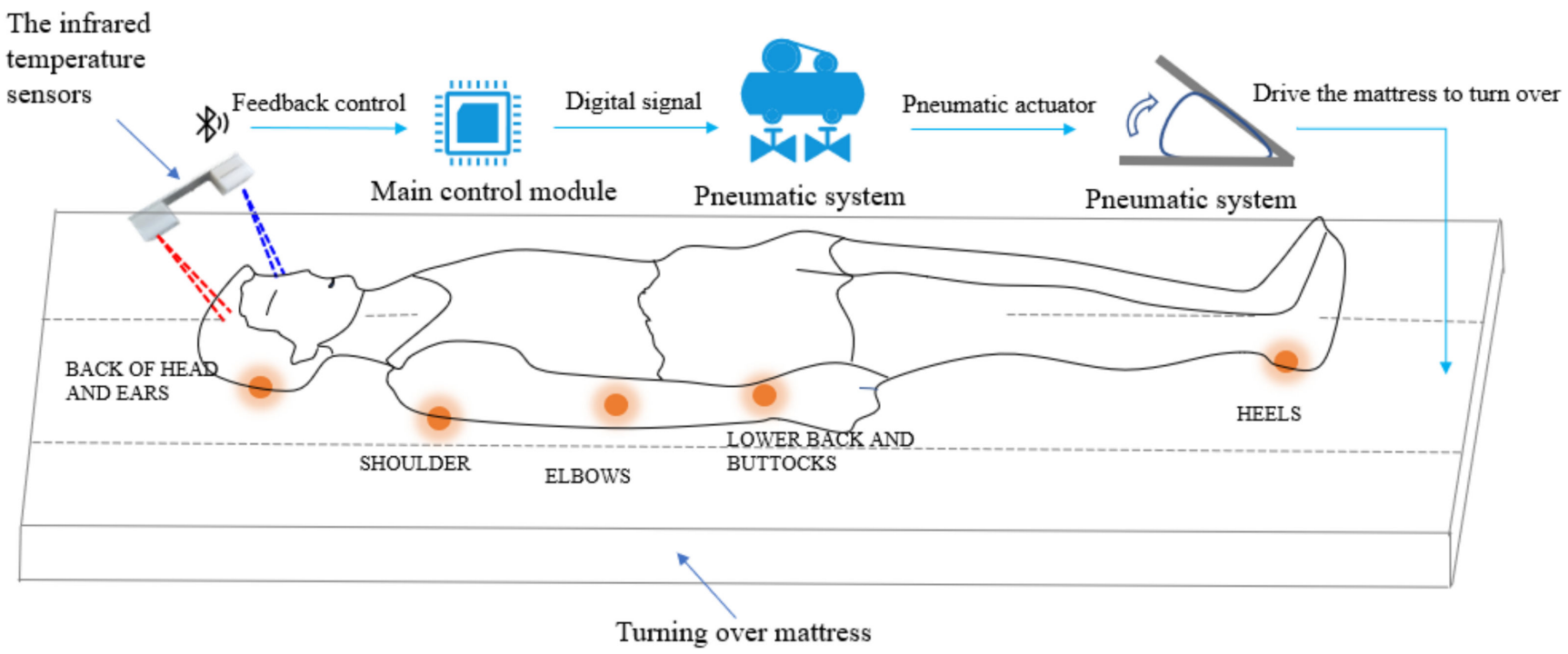

Figure 1. Human body turnover process monitoring and controlling system.

\section{Materials and Methods}

\subsection{Turning-Body over Methods}

Assisted turning over is an important part of nursing care for long-term bedridden patients. Clinically, the following nursing steps are specified: (1) Let the patient lie on his back, place his hands on his chest and bend his legs. (2) Move the patient's lower limbs to the edge of the nurse's bed and then move the patient's shoulders out. (3) Hold the shoulders with one hand and knees with the other hand and push the patient to the opposite side so that the patient faces the nurse [16].

After turning over, cushion the patient's back and limbs with pillows to make the patient comfortable and safe. Although this approach is mature and practical, the amount of effort required to interact with the care recipient can be better determined. In addition, the consistency of artificial care force is poor, which is easy to cause body deviation, shear stress and rotation stress, and cause secondary damage to the body. For nursing equipment, it is difficult to stably apply turning thrust due to the self-bending nature of the knee joint. Considering good support and achievability, our design uses two support points to complete the turning action, and one of the stress points is adjusted to the hip joint at the same time. The turning over method before and after improvement is shown in Figure 2. 


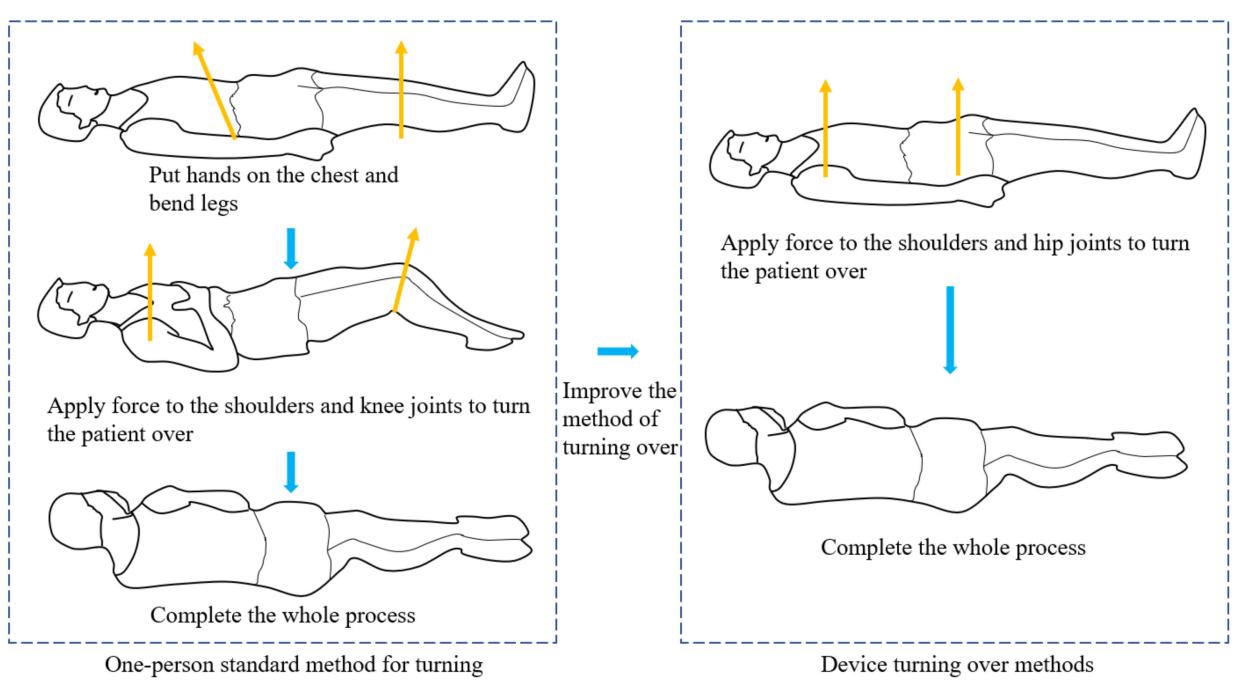

Figure 2. Schematic diagram of one-person standard and device turning over methods.

Human-computer interaction equipment often has higher requirements for safety. Various sophisticated mechanical arms in the industry have been quite mature and have stable control, but various types of robots that interact with humans are often still in the research and development test stage. At this stage, it is difficult to achieve refined care for people through equipment. Based on the premise of solving practical problems, we use flexible airbags to drive the mattress to realize the automatic turning function of the bedridden elderly, so as to reduce the working pressure of nurses and reduce the incidence of PU.

\subsection{Mathematical Model of the Soft Actuator}

The actuator is an irregular airbag made of thermoplastic polyurethanes, which pushes the mattress to rotate through expansion force. As shown in Figure 3, it is a simple model of extension and case. Suppose that part of the airbag is pressed on the board, and the rest is stretched between the partitions from the separation point; in order to obtain the shape of the airbag inflated and expanded, the value at the maximum volume is taken.
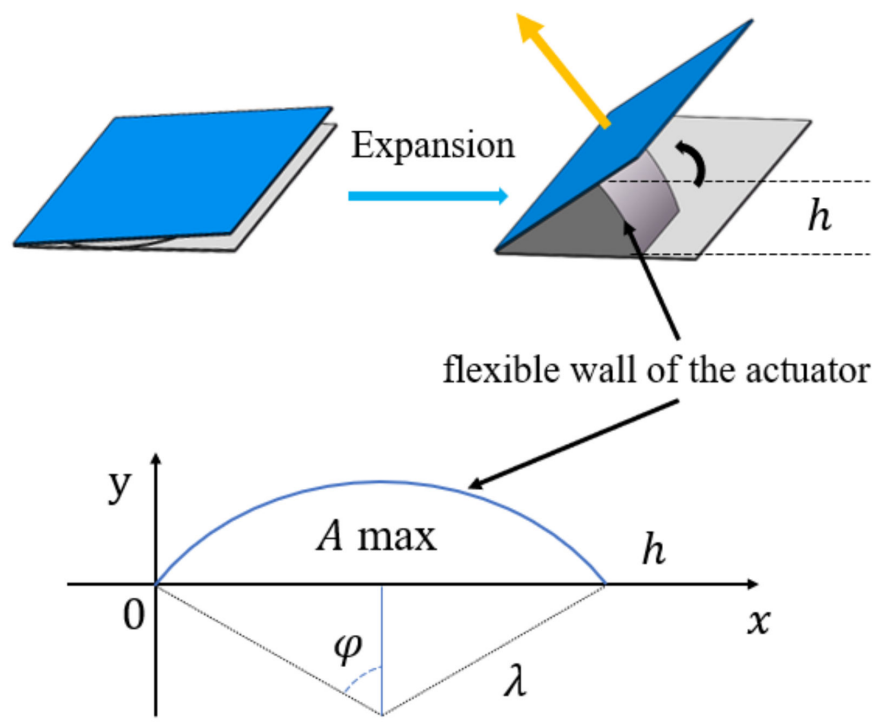

Arc of a circle as maximum area

Figure 3. A simple soft actuator on the expansion principle. 
Assuming that the membrane of the airbag forms an arc, the equation about the arc radius $\lambda$ is obtained by solving the boundary value problem through the Lagrangian multipliers [26]:

$$
\lambda^{2}=\left(x-\frac{h}{2}\right)^{2}+\left(y+\sqrt{\lambda^{2}-\frac{h^{2}}{4}}\right)^{2}
$$

Based on this equation, we can calculate the maximum boundary of airbag deformation to help us design the size of the airbag protective sleeve. The protective cover can fix the airbag to the design area of the mattress. In order to achieve good support, the shape of the turning airbag is designed as a wedge, and the size of the airbag is limited in order to reduce the inflation time. The airbag driving force can be calculated as the product of the area of the contact plate and the internal pressure $P$. Combining the length $L$, width $b$ and height $h$ of the actuator element between the plates, the following formula gives the mathematical model used to describe the relationship of the driving force $F$, the internal pressure $P$ and contact area [26]:

$$
F=P\left[\frac{\pi}{4}\left(L-\frac{1}{2} \pi \cdot h\right)^{2}+(b-L) \cdot\left(L-\frac{1}{2} \pi \cdot h\right)\right]
$$

The above equation can help us confirm the design pressure, which can carry out gas path design and related component selection.

\subsection{Pneumatic Turnover Mattress}

It is believed that turning over regularly can effectively improve the pressure distribution of bedridden patients and reduce the harm of positive pressure to areas with high incidence of PU. Shear force is another cause of PU [27]. Shear force is applied to the surface of adjacent objects, causing progressive parallel sliding force in the opposite direction. It acts on the deep layers of the skin, causing relative tissue displacement and cutting off the blood supply to a larger area. Therefore, shear force is more harmful than vertical pressure [28]. Combining the above method, as shown in Figure 4, we design a pneumatic turning mattress that can turn over and reduce the harm to the body by shearing force. The mattress is divided into three parts, the left and right sides are the supporting areas, and the middle is the turning area. The normal palm foam mattress is in direct contact with the patient's body, and the airbags are attached under the mattress, and the airbags in the two areas work together to achieve turnover [29].

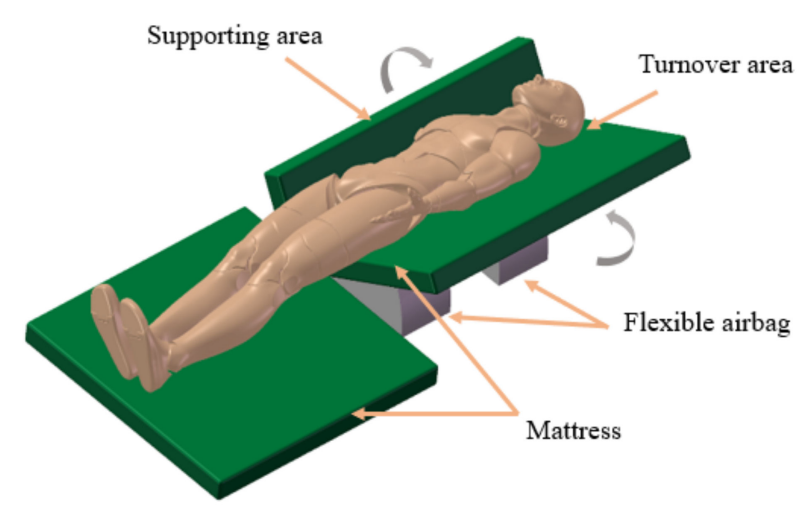

Figure 4. Schematic diagram of the mattress.

As shown in Figure 5, when the body is tilted, the supporting force of the support airbag acting on the human body reduces the friction. When turning over, airbag a is first inflated to prop up OA rotating from horizontal to $90^{\circ}$. Then airbag $b$ is inflated to prop up $\mathrm{OB}$ rotating to a certain angle (between $30^{\circ}$ and $45^{\circ}$, which can be changed according to patient comfort). At the same time, airbag a slowly deflates, keeping $\angle \mathrm{AOB}$ at about 
$90^{\circ}$ to complete the entire turning operation. In entire turning operation process, $\angle \mathrm{AOB}$ is kept at about $90^{\circ}$ and the airbag a supports the side of the human body to give thrust $\mathrm{F}$ to reduce the frictional force $\mathrm{f}$ to reduce the local shear force generated by the body position change and improve the comfort of the turnover process. The whole turning over process is divided into three steps, as shown in Figure 6.

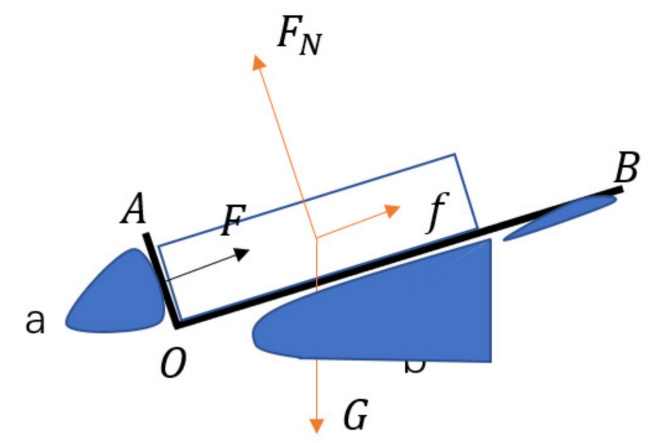

Figure 5. Schematic diagram of driving process analysis.

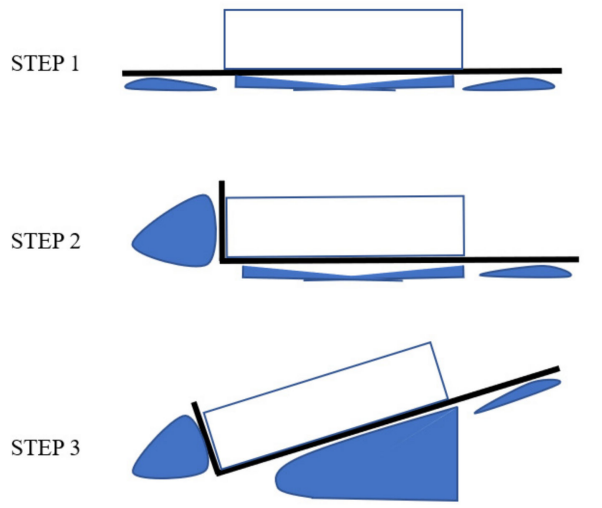

Figure 6. Three steps in the turnover process.

\subsection{Design of Control System}

The schematic diagram of the air circuit is shown in Figure 7. When the three-way valve is de-energized, $1-3$ is on and 1-2 is off; when it is energized, $1-2$ is on and 1-3 is off. When turning over on the left side, supporting airbags need to be inflated at first, then valve $a$ and valve $c$ are energized, and valve $b$ is de-energized. Gas can be rushed into the airbags from the outside through the air pump; when the airbags are deflated, valve a is de-energized and valve $b$ and valve $c$ are energized. The air pump can draw air out of the one airbag.
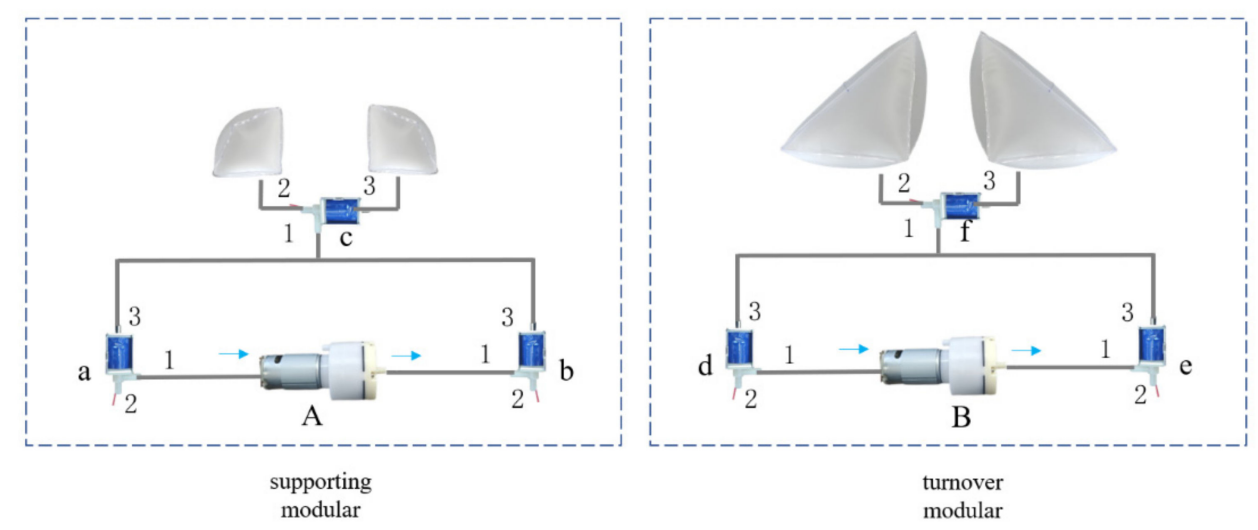

Figure 7. Schematic diagram of gas circuit. 
When turning over on the right side, supporting airbags need to be inflated at first, then valve $a$ is energized, and valve $b$ and valve $c$ are de-energized. Gas can be rushed into the airbags from the outside through the air pump; when the airbags are deflated, valve a and valve $c$ are de-energized and valve $b$ is energized. The air pump can draw air out of the airbags.

The entire system is contturned by the ESP32, and t each valve and the pump are contturned through the relay module. During the whole turning motion process, air pump B has been running at a constant power, while air pump A dynamically changes the power to keep the angle between the support surface and the turning surface at about $90^{\circ}$, and air pump A is individually contturned by a depressurization module through a single-chip microcomputer. The output analog quantity controls the output voltage. The system uses XGZP6857 gas pressure sensor module to monitor the gas pressure.

\subsection{Design of Temperature Monitoring System}

\subsubsection{Design of Infrared Temperature Sensor}

The infrared temperature sensor is based on the Seebeck effect, and its working principle is shown in Figure 8. When external infrared radiation irradiates the absorption area of the detector, the absorption area absorbs the infrared radiation and converts it into heat energy. Since the hot junction area at the connected end of the thermocouple and the cold junction area at the open end of the thermocouple are thermally isolated, a temperature gradient will be generated in the hot junction area and the cold junction area. The temperature gradient can be converted into a voltage signal output $\left(V_{\text {out }}\right)$ through the Seebeck effect of the thermocouple material.

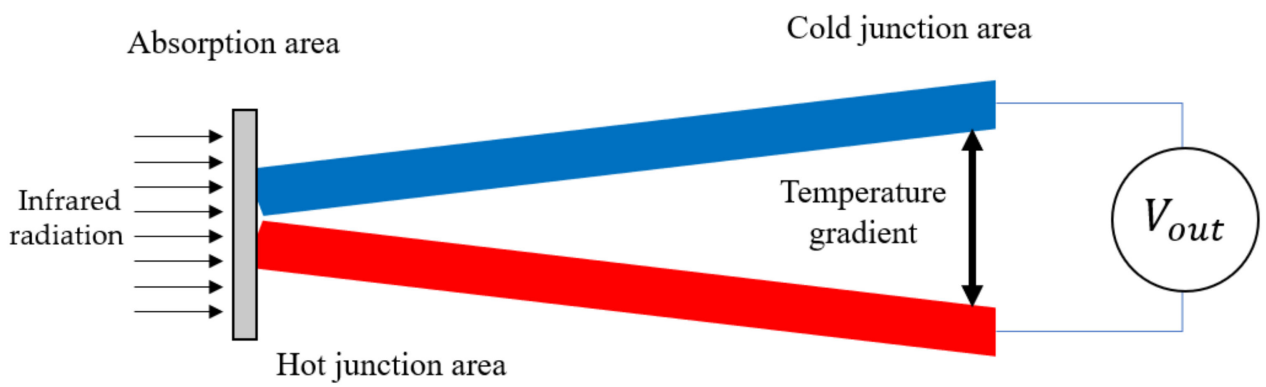

Figure 8. Schematic diagram of Seebeck effect principle.

The non-contact infrared temperature sensor uses the technology of Micro-ElectroMechanical System (MEMS) to process two different materials into thermocouples. The thermopile structure is realized on the sensor. In order to enhance the signal, $\mathrm{N}$ pairs of thermocouples are usually connected in series. The output voltage of the non-contact MEMS infrared temperature sensor can be expressed as:

$$
V_{\text {out }}=N\left(S_{A}-S_{B}\right)\left(T_{H}-T_{C}\right)=N S_{A B} \Delta S T_{H C}
$$

where $N$ is the logarithm of the thermocouple, $S_{A}$ and $S_{B}$ are the Seebeck coefficients of two materials, $T_{H}$ and $T_{C}$ are the temperature of the hot end and the cold end.

The structure diagram of non-contact MEMS infrared temperature sensor is shown in Figure 9. The optical system aggregates the target infrared radiation energy in the receiving hole, and the size of the field of view (FOV) is determined by the optical parts of the sensor and its position. An application-specific integrated circuit (ASIC) is integrated in the sensor, which plays the role of measuring ambient temperature and digital processing of electrical signals. Infrared energy focuses on the MEMS thermopile and transforms into the corresponding electrical signal. This signal is processed by a signal amplifier, an AD converter, and a signal processor. The internal algorithm and the target emission rate are changed to the temperature value of the target. Signal flow chart is shown in Figure 10. 


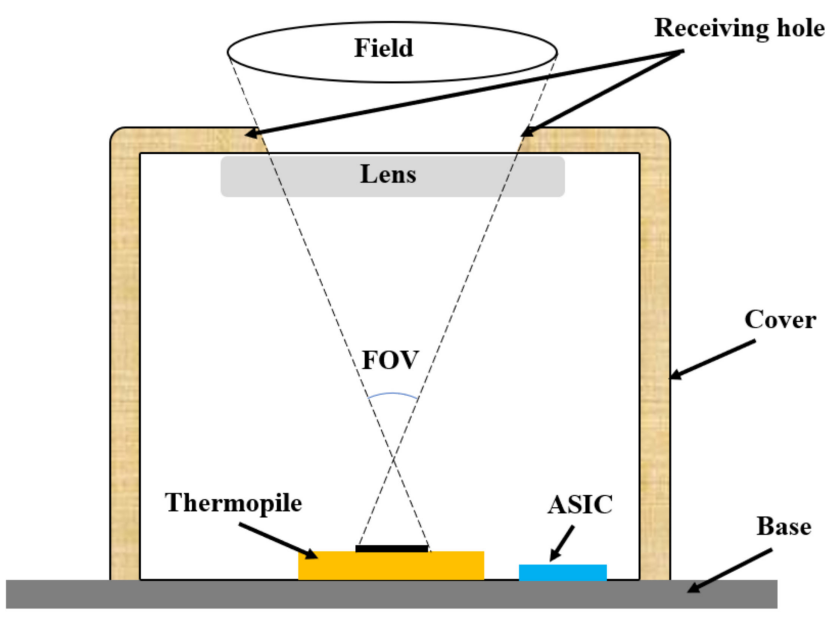

Figure 9. Schematic diagram of infrared temperature sensor.

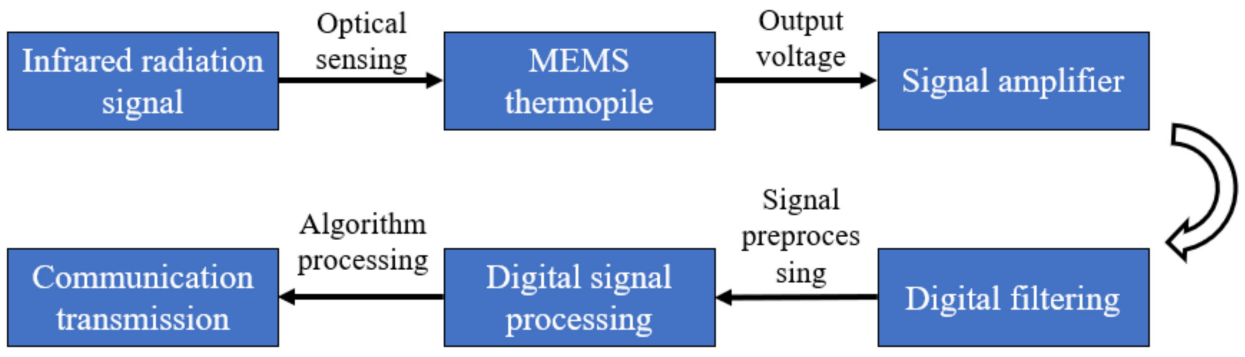

Figure 10. Signal flow chart.

\subsubsection{Design of Sensor Placement}

The infrared temperature sensor is used for real-time temperature measurement, position monitoring, and turning over monitoring of bedridden patients. According to the requirements of using nursing mattresses, the elderly should lie on the $40 \mathrm{~cm}$ wide turnover area of the mattress. According to the actual situation, allow the patient's body to lean by $8 \mathrm{~cm}$ and the head to rotate $25^{\circ}$. The orange head outline in Figure 11 is the area where the head is upright and the head rotates $25^{\circ}$ when lying in a normal position. The green rectangle is the area where the head is up and the head rotates $25^{\circ}$ when the side is $8 \mathrm{~cm}$. The overlapping area of the four positions is filled with red, taking the overlapping area The forehead is used as the target measurement area (yellow circle in the figure), which corresponds to the upper side of the patient's eyebrows. The same is true on the other side. Two infrared temperature sensors are used to collect the facial temperature data of the elderly in real time.

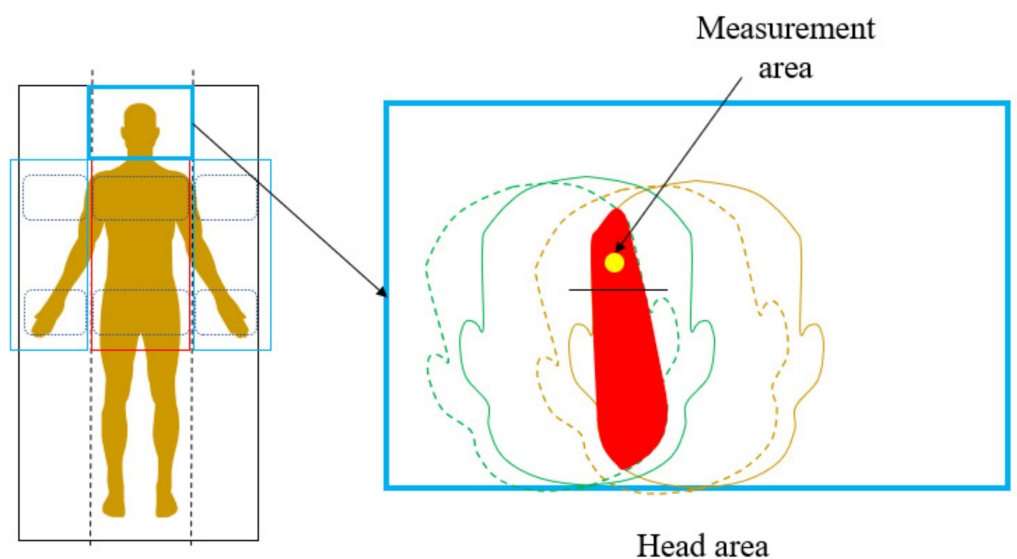

Figure 11. Schematic diagram of temperature sensor measurement area. 
The measurement distance directly affects the measurement accuracy. As shown in Figure 12, the measured object in position one is far away and does not completely fill the temperature measurement field of view, and there is background radiation interference; in position two the measured object exactly fills the field of view, which is the maximum position for accurate measurement; position three has no background. Radiation interference is an accurate measurement location. The measurement distance of the infrared temperature sensor is related to the field angle of the infrared probe. In order to increase the measurement distance and reduce the interference of background radiation energy, lens focusing is used to reduce the field angle of the infrared probe, which is $-7.5^{\circ}$ to $7.5^{\circ}$. The distance coefficient value is the ratio of the measured distance $\mathrm{D}$ to the diameter $\mathrm{S}$ of the sensor measurement area under this distance, and the distance coefficient of the probe is calculated according to the field of view.

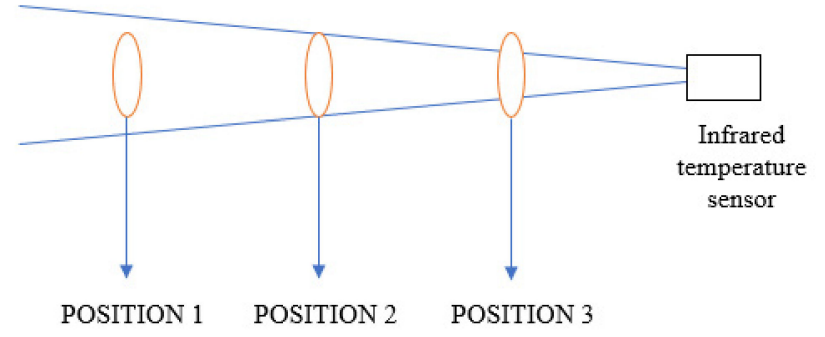

Figure 12. Schematic diagram of the relationship between the measured object and the field of view of the infrared temperature measurement system.

The temperature sensing device is placed as shown in Figure 13. According to the calculated measurement distance and range, it is placed at a suitable position on the head of the bed, and the field of view on both sides is within the set area. It can cover the reasonable movement range of the head and carry out real-time forehead temperature monitoring.

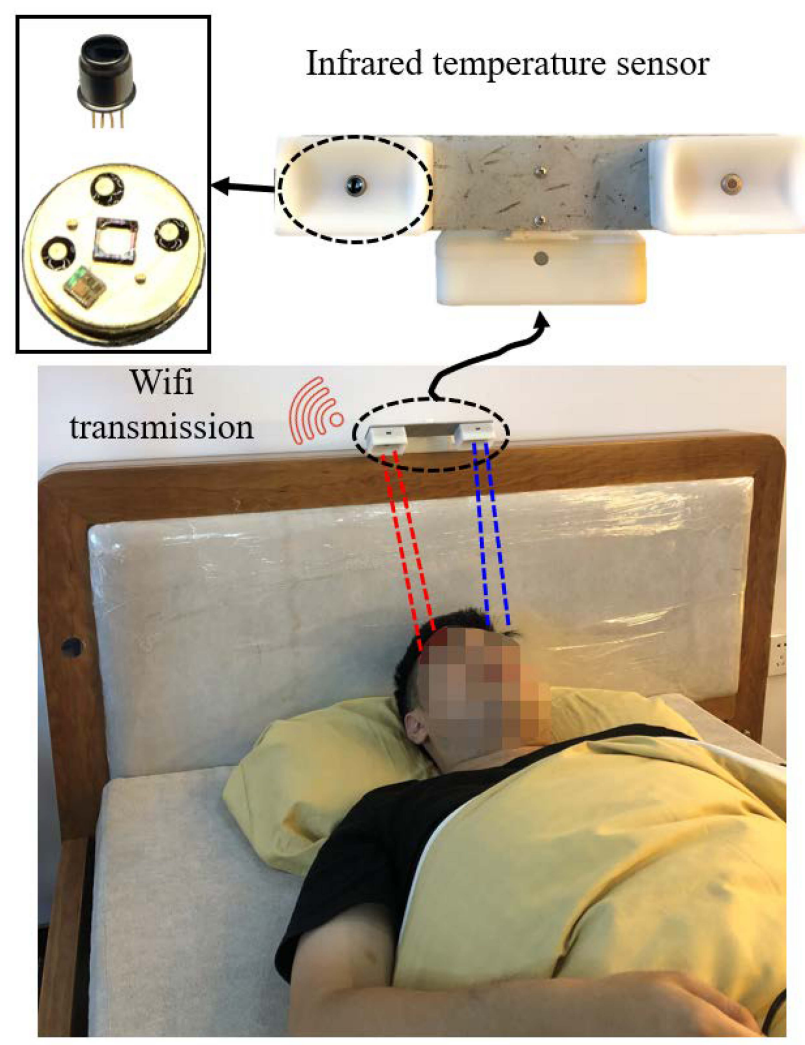

Figure 13. Schematic diagram of temperature sensing device monitoring. 


\section{Results}

\subsection{Mattress Turnover Test}

The experimenter imitated the disabled elderly lying on a mattress. First, the support airbags were inflated with a constant maximum power. After about $50 \mathrm{~s}$, the support surface reached a predetermined angle. Then turning airbags started to inflate with a constant maximum power, and the support airbags began to deflate with dynamic changes. After about $150 \mathrm{~s}$, the supporting surface and the turning surface reached a predetermined angle, as is shown in Figure 14. The value of the entire pressure sensor is much smaller than the maximum pressure resistance of the airbags, so the subsequent work does not monitor the air pressure. According to the feedback of the experimenter, the comfort of the pneumatic turnover mattress is better than that of the conventional one-side turnover bed.

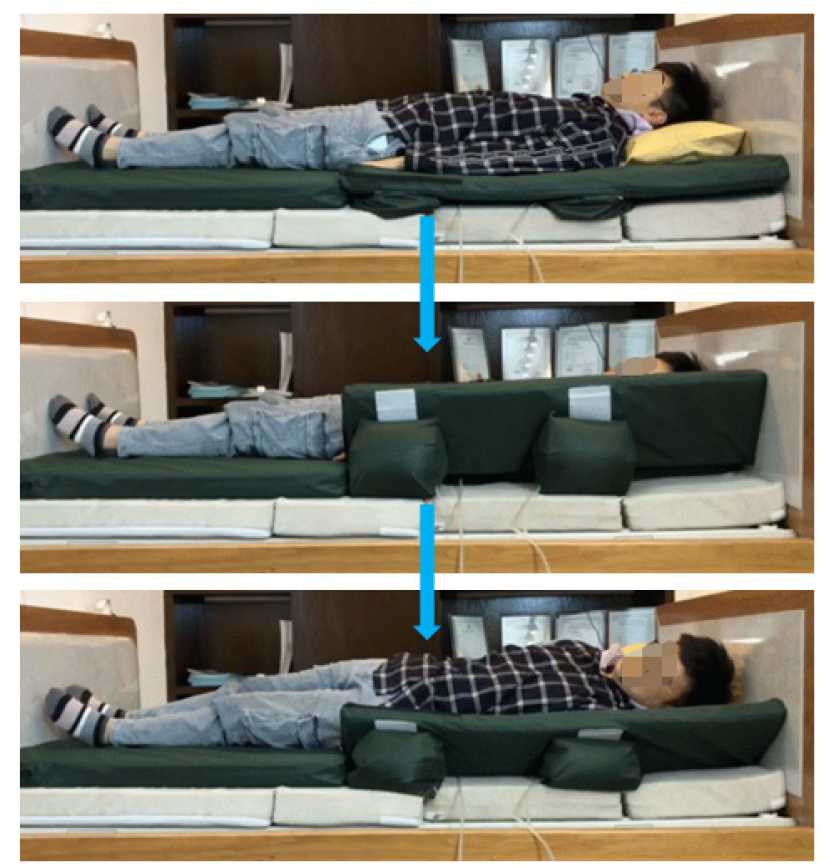

Figure 14. Three steps in the process of turnover.

\subsection{Temperature Sensor Monitors the State of Bed Rest}

\subsubsection{Real-Time Forehead Temperature Measurement}

The sensor we use uses a Negative Temperature Coefficient (NTC) thermistor as ambient temperature compensation. When the patient is not turning over, take the temperature of the patient's forehead. Ambient temperature is an important factor in infrared temperature measurement. The output voltage of the thermopile is different under different ambient temperatures. In this scenario, the accuracy requirements are high. Therefore, we calibrate the temperature sensor. The calibration of the sensor needs to provide two conditions: one is to provide an accurate ambient temperature for the sensor; the other is to provide a standard target temperature.

We have customized a set of fixtures and assembled the sensor on the fixtures. The fixtures are filled with circulating water at a set temperature, which is controlled by a thermostatic water tank. The advantage of this is that the temperature control is accurate and is not affected by the environment in which the experimental equipment is located. Another thermostatic water tank equipped with a standard blackbody provides the target temperature. The blackbody penetrates in the water tank, and the temperature of the blackbody can be adjusted by controlling the temperature of the water tank. We aligned the fixture with the sensor to the black body and measured and recorded the value of the temperature sensor. The calibration equipment is shown in Figure 15. 


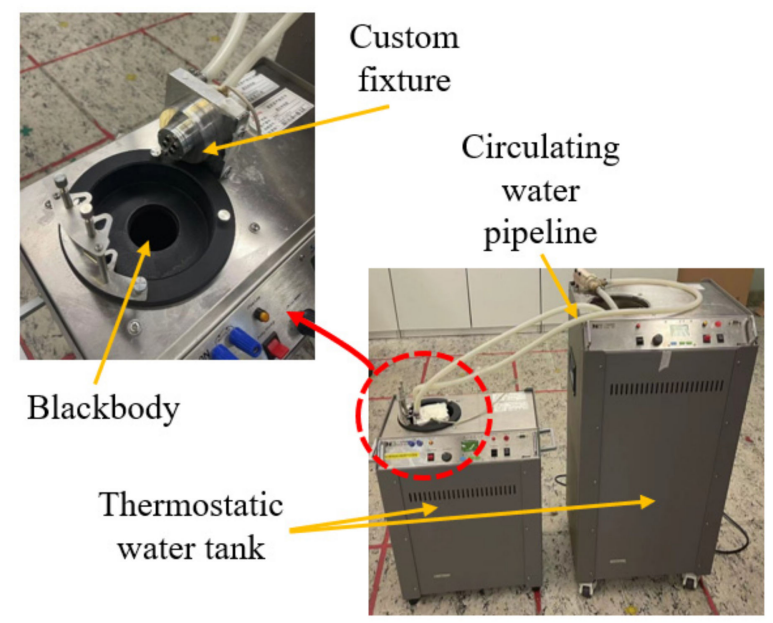

Figure 15. Temperature sensor calibration tool diagram.

In order to improve the measurement accuracy, the measured value and the true value of the thermopile output voltage and the ambient temperature were obtained through the calibration experiment, and then the segmented interpolation was used for temperature compensation to obtain a relatively accurate measurement value. The larger value measured by the two sensors is the final measured temperature.

As shown in Figure 16, at a room temperature of about $28^{\circ} \mathrm{C}$, the sensor was used to record the temperature data of the experimenter once per second for $20 \mathrm{~s}$. The temperature measured by the medical forehead gun is $36.7^{\circ} \mathrm{C}$, the average temperature measured by the sensor is $36.41{ }^{\circ} \mathrm{C}$, and the temperature difference is about $0.3{ }^{\circ} \mathrm{C}$, which has clinical significance.

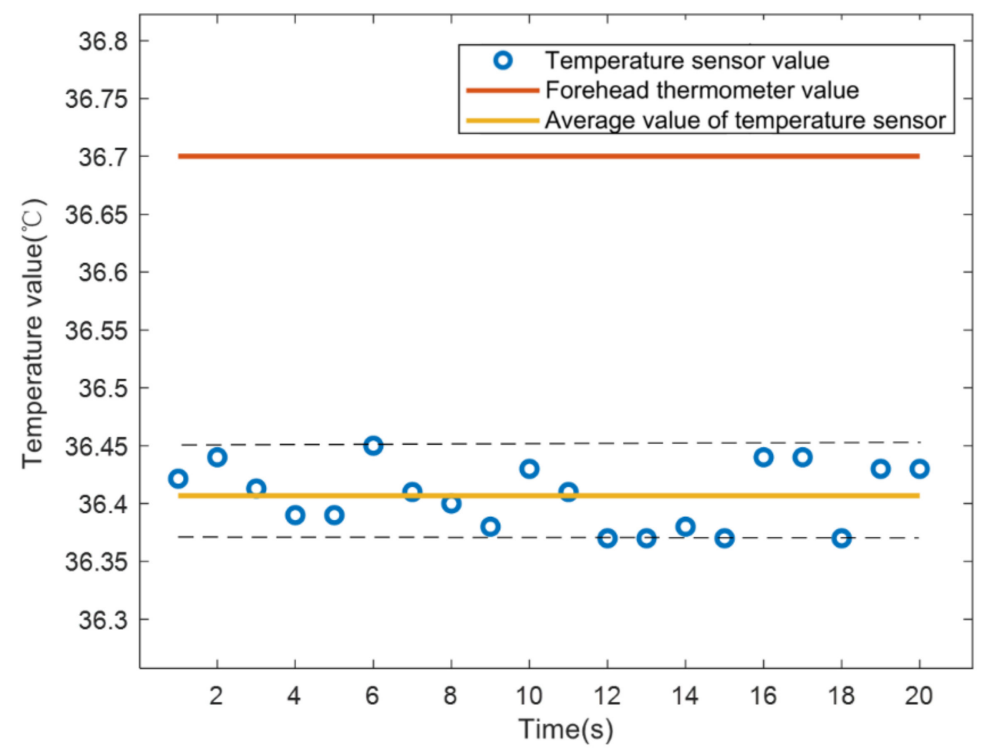

Figure 16. Comparison of temperature measurement between infrared temperature sensor and medical forehead thermometer.

\subsubsection{Out-of-Bed Monitoring and Position Monitoring}

Turning over this mattress is the core function. The airbag drives the mattress to tilt the patient's body by about $30-45^{\circ}$, and the bedridden patient can automatically complete the turning over without the cooperation of the nurse, so as to improve the compression area of the body to prevent PU. This requires that the patient must lie in a prescribed position. The temperature sensor is now used for threshold monitoring to identify the position of the patient on the bed. 
We use an inertial measurement unit (IMU) to measure the head swing angle, and at the same time use the left and right sensors to record changes in forehead temperature. As shown in Figure 17, the head is in the middle position from 0 to $2 \mathrm{~s}$, and both sensors can collect facial temperature data, the value is above $250 \mu \mathrm{V} ; 3$ to $5 \mathrm{~s}$, the head is offset to the right by about $30^{\circ}$, and the measurement area of the left sensor is Mattress pillow, the value is about $175 \mu \mathrm{V} ; 5$ to $7 \mathrm{~s}$, the head returns to the center position; 7 to $9 \mathrm{~s}$, the head is about $30^{\circ}$ to the left, at this time the right sensor measuring area is the pillow, the value is about $180 \mu \mathrm{V} ; 9$ to $11 \mathrm{~s}$, the head is left Offset $50^{\circ}$, at this time the face is completely out of the measurement range, the value is about $170 \mu \mathrm{V}$.

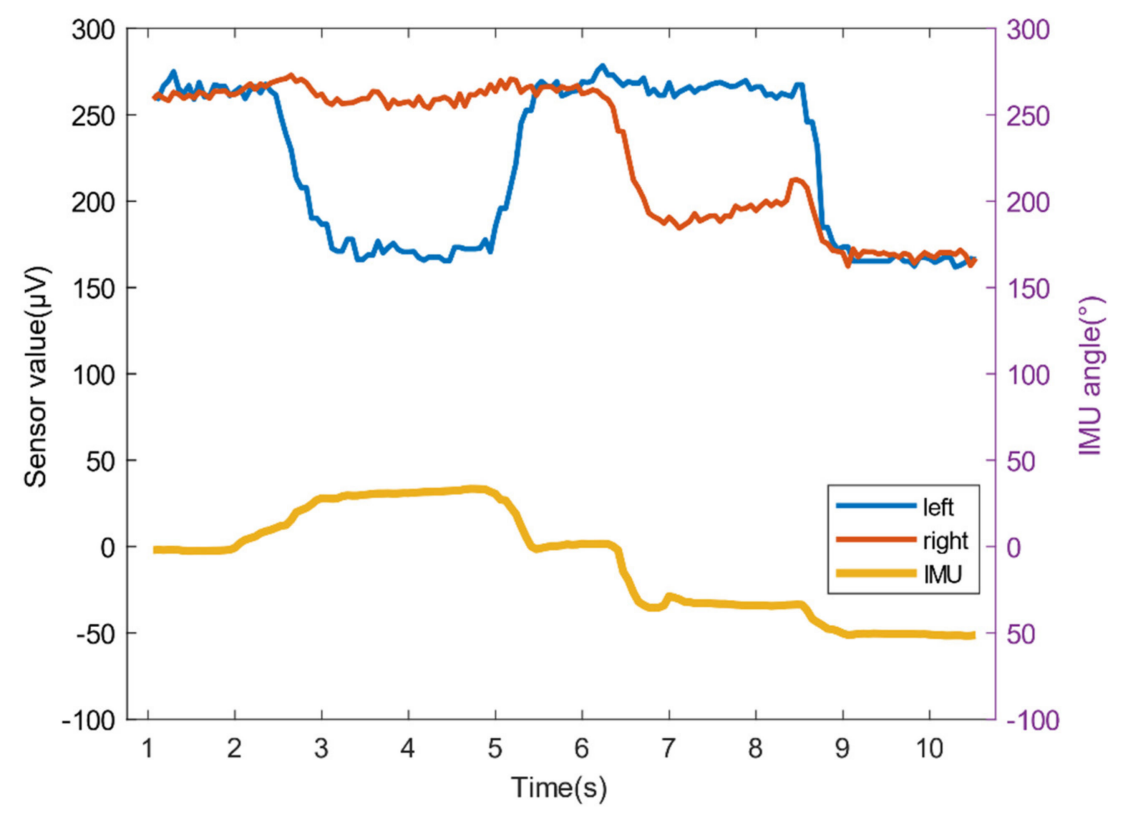

Figure 17. The relationship between the head swing angle and the sensor value change.

When the values of the left and right sensors are less than the threshold value for more than a certain period of time, the elderly may be in an improper position or even get out of bed, and an alarm will be activated; when the voltage value is greater than or equal to the threshold value, it shows that the elderly position is normal and can be turned over through mattress.

\subsubsection{Real-Time Monitoring of the Turning Process}

Turning over is the process of placing the patient's body at an angle, which is unpredictable. The infrared temperature sensor monitors the change of the thermopile output voltage value during the patient's turning over to realize the monitoring of the whole process. When the monitoring value is abnormal, the system will immediately stop turning and give an alarm.

In the experiment, we asked the experimenter to lie in the middle, left, and right of the mattress (the offset distance is $8 \mathrm{~cm}$ ), and use the left and right sensors above the forehead to measure the temperature data during the entire turning-flattening process, and an IMU was used to record the changes in the inclination angle of the experimenter's body during the turnover process in real time.

As shown in Figure 18, the temperature value and angle value trend can be captured by the sensor value changes at the three state nodes of the beginning and end to ensure that the experimenter completes the entire turn over care process through the mattress. 


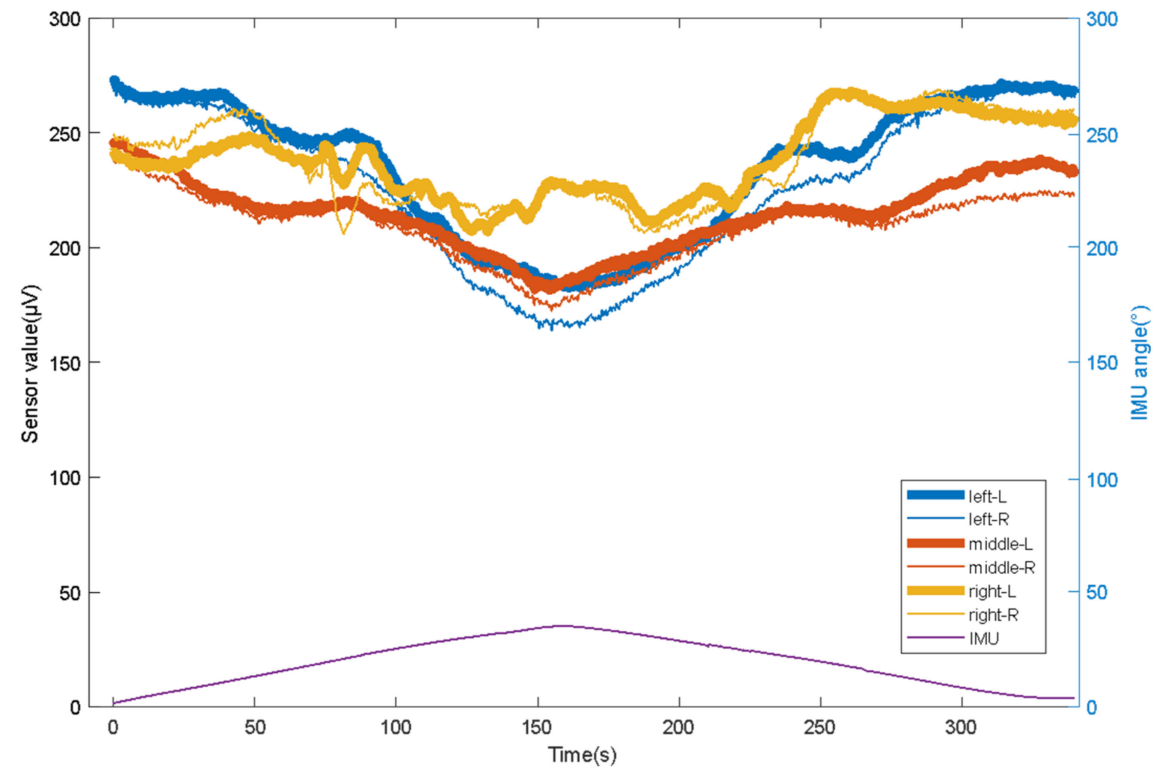

Figure 18. The relationship between the head swing angle and the sensor value change.

Figure 19 shows the detection values of the whole process of the left turning over of the mattress when the experimenter lies in the middle, left and right of the mattress, and when there was no one on the bed. When the experimenter lies in the middle position, with the increase of the left turning angle of the mattress, the right sensor loses the human body temperature. At this time, its value is close to that when there is no one, and the value of the left sensor is always more than $20 \%$ higher than that when there is no one. When the experimenter lies in the left leaning position, with the increase of the left inclination angle of the mattress, there is a loss of human body temperature on the right side. At this time, its value is close to that when there is no one, and the value of the left sensor is always more than $10 \%$ higher than that when there is no one. When the experimenter lies in the right deviation position, the left and right sensor values are always more than $20 \%$ higher than when there are no patients. Therefore, the temperature sensor can clearly distinguish whether there are people in the measurement area, which can alarm in time when the patient falls into bed and other accidents.

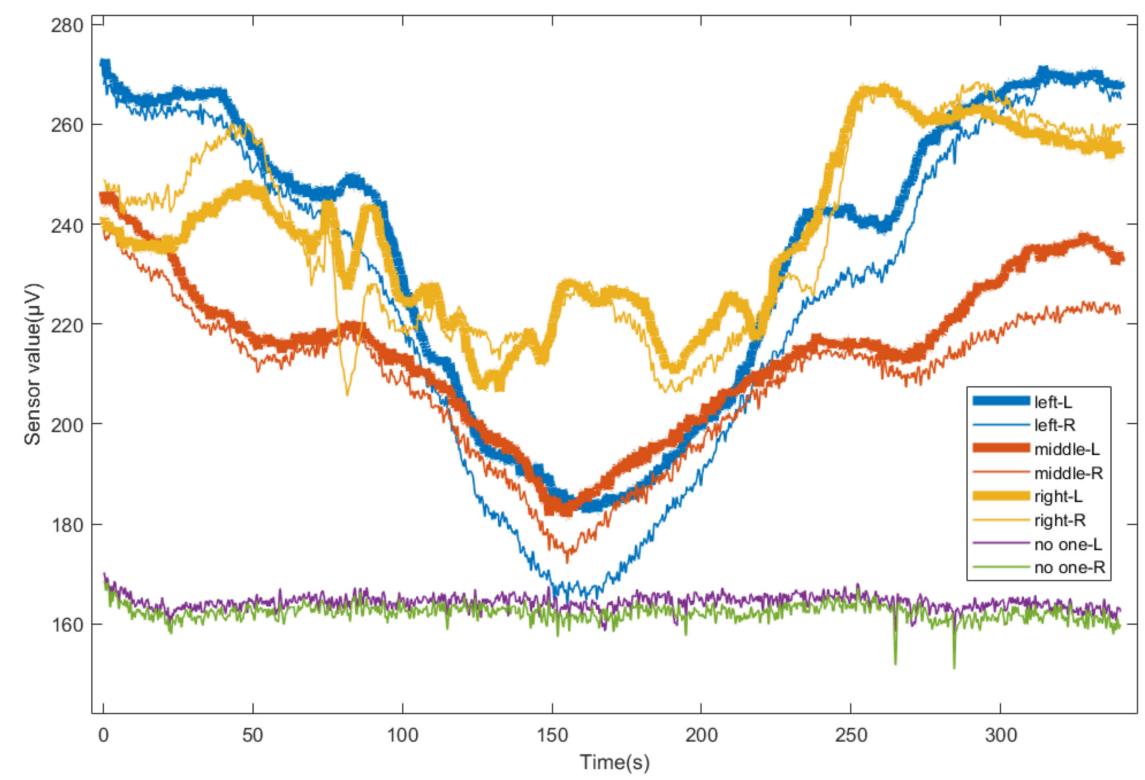

Figure 19. Change of sensor value during turning down in different positions. 


\section{Conclusions and Discussion}

We have developed a flexible turning and sensing system for disabled patients, which includes a pneumatic turning mattress and a temperature monitoring device. The turnover mattress uses turning airbags and supporting airbags to reduce the shearing force generated in the body part when the body position changes, and achieve a better PU prevention effect. The system uses a near-infrared temperature sensor to monitor the status of a patient lying in bed, ensuring that the patient lies in a suitable position to automatically turn over through the mattress. The temperature sensor is also used to measure the patient's temperature in real time. At the same time, the patient's bed state and automatic turning state can be monitored in real time, which can set off an alarm when getting out of bed or improperly lying. The infrared temperature sensor can monitor the position status of the elderly in real time during turning over. When the monitoring value is in the abnormal range, it can stop the air pump and start the alarm in time.

We have verified the functionality of the automatic turnover system through experiments. In the future, we hope that it can help enough people solve problems, including reducing the work intensity of nurses and improving the comfort of turning over nursing. We will also conduct research in the following directions:

(1) To adapt to more patients. When designing the mattress, we considered that the turnover system should meet most middle-aged and elderly patients' body width. For this reason, we refer to GB10000-80 (Chinese adult human body size measurement data) to design the width of the turnover area reasonably. The angle between the turning surface and the support surface is also an important factor that affects the experience. We plan to change the angle to compensate for the comfort of patients with different body widths to provide good support and reduce the occurrence of body tilt friction force. If the shoulder width of the patient is larger than the width of the turning area we designed, the turning angle of the side support surface can be reduced to give consideration to comfort. Similarly, if the shoulder width is greater than the width of our designed turning area, the turning angle of the side support surface can be increased to provide better support. On the premise of ensuring a certain turning angle, the corresponding relationship between different shoulder widths and included angles is found through the subjective evaluation of comfort by the experimenter. If the results are difficult to classify, we will consider allowing patients to adjust a comfortable angle as a necessary parameter for each automatic turning.

(2) To improve the accuracy of temperature monitoring. The temperature sensor monitoring device is an important part of our system. We want to use it to realize realtime temperature measurement, out-of-bed alarm, and turn-over status monitoring of bedridden patients. Since the measurement environment is relatively complex, the uncertainty of the measurement area affects the accuracy of the measurement value, and it is difficult to improve the measurement accuracy through conventional compensation methods. Ambient temperature is the key reference data for measuring object temperature. The temperature of background items such as pillows and bedding is also different under different ambient temperatures. We need to measure the temperature of background items under different ambient temperatures as reference values. When the value of the sensor at a specific temperature is significantly higher than the temperature of the background object, it is determined that the patient is detected within the measurement range; otherwise, it is determined that there is no one. In our design, the position of sensor illumination is very important, which will directly affect the accuracy of the sensing system. In order to improve the positioning accuracy, we plan to add several ray sources around the sensor to assist the positioning.

Author Contributions: T.L. and X.Z. (Xiaofeng Zou); methodology, Y.Z.; software, X.Z. (Xiaofeng Zou); validation, B.Z.; formal analysis, B.Z.; investigation, Y.Z. and Y.H.; resources, S.W. and X.Z. (Xiufeng Zhang); data curation, X.Z. (Xiaofeng Zou); writing-original draft preparation, X.Z. (Xiaofeng Zou); writing - review and editing, X.Z. (Xiaofeng Zou); visualization, X.Z. (Xiaofeng Zou); supervision, T.L.; project administration, Y.H.; funding acquisition, X.Z. (Xiufeng Zhang). All authors have read and agreed to the published version of the manuscript. 
Funding: This work was supported in part by the National Natural Science Foundation of China under 52175033, 51775485 and U1913601; the Zhejiang Provincial Natural Science Foundation of China under Grant No. LZ20E050002.

Informed Consent Statement: Informed consent was obtained from all subjects involved in the study.

Conflicts of Interest: The authors declare no conflict of interest.

\section{References}

1. Gaspar, S.; Peralta, M.; Marques, A.; Budri, A.; De Matos, M.G. Effectiveness on hospital-acquired pressure ulcers prevention: A systematic review. Int. Wound J. 2019, 16, 1087-1102. [CrossRef]

2. Serraes, B.; Van Leen, M.; Schols, J.; Van Hecke, A.; Verhaeghe, S.; Beeckman, D. Prevention of pressure ulcers with a static air support surface: A systematic review. Int. Wound J. 2018, 15, 333-343. [CrossRef] [PubMed]

3. Li, T.; Wang, L.; Yi, J.A.F.T.; Li, Q.; Liu, T. Reconstructing Walking Dynamics from Two Shank-Mounted Inertial Measurement Units (IMUs). IEEE/ASME Trans. Mechatron. 2021, 1. [CrossRef]

4. Zhong, Z.; Chen, F.; Zhai, Q.; Fu, Z.; Ferreira, J.; Liu, Y.; Yi, J.; Liu, T. A real-time pre-impact fall detection and protection system. In Proceedings of the 2018 IEEE/ASME International Conference on Advanced Intelligent Mechatronics (AIM), Auckland, New Zealand, 9-12 June 2018; pp. 1039-1044.

5. Nageswaran, S.; Vijayakumar, R.; Sivarasu, S. Design of mechanical interface to re-distribute excess pressure to prevent the formation of decubitus ulcers in bed ridden patients. In Proceedings of the 2015 37th Annual International Conference of the IEEE Engineering in Medicine and Biology Society (EMBC), Milan, Italy, 25-29 August 2015; pp. 1021-1024.

6. Demarré, L.; Van Lancker, A.; Van Hecke, A.; Verhaeghe, S.; Grypdonck, M.; Lemey, J.; Annemans, L.; Beeckman, D. The cost of prevention and treatment of pressure ulcers: A systematic review. Int. J. Nurs. Stud. 2015, 52, 1754-1774. [CrossRef] [PubMed]

7. Nair, P.; Mathur, S.; Bhandare, R.; Narayanan, G. Bed sore Prevention using Pneumatic controls. In Proceedings of the 2020 IEEE International Conference on Electronics, Computing and Communication Technologies (CONECCT), Bangalore, India, 2-4 July 2020; pp. 1-5.

8. Guo, S.; Kato, Y.; Ito, H.; Mukai, T. Development of rubber-based flexible sensor sheet for care-related apparatus. SEI Tech. Rev. 2012, 75, 125-131.

9. Meaume, S.; Marty, M. Pressure ulcer prevention using an alternating-pressure mattress overlay: The MATCARP project. J. Wound Care 2020, 29 (Suppl. 9a), S32-S38. [CrossRef]

10. Misaki, A.; Imanishi, K.; Takasugi, S.I.; Wada, M.; Fukagawa, S.; Furue, M. Body pressure sensing mattress for bedsore prevention. SEI Tech. Rev. 2014, 79, 95-99.

11. Aleksandrowicz, H.; Owczarczyk-Saczonek, A.; Placek, W. Venous Leg Ulcers: Advanced Therapies and New Technologies. Biomedicines 2021, 9, 1569. [CrossRef] [PubMed]

12. Tayyib, N.; Coyer, F. Effectiveness of pressure ulcer prevention strategies for adult patients in intensive care units: A systematic review. Worldviews Evid.-Based Nurs. 2016, 13, 432-444. [CrossRef]

13. Bergstrom, N. Treatment of Pressure Ulcers: Clinical Practice Guideline; DIANE Publishing: Collingdale, PA, USA, 2004.

14. Carter, M.J. Economic Evaluations of Guideline-Based or Strategic Interventions for the Prevention or Treatment of Chronic Wounds. Appl. Health Econ. Health Policy 2014, 12, 373-389. [CrossRef]

15. Hayakawa, T.; Misaki, A.; Takasugi, S.I.; Furue, M. Smart Rubber Active Mattress" Taiatsu Bunsan. SEI Tech. Rev. 2017, 85, 65-67.

16. Yousefi, R.; Ostadabbas, S.; Faezipour, M.; Nourani, M.; Ng, V.; Tamil, L.S.; Bowling, A.; Behan, D.; Pompeo, M. A smart bed platform for monitoring \& ulcer prevention. In Proceedings of the 2011 th International Conference on Biomedical Engineering and Informatics (BMEI), Shanghai, China, 15-17 October 2011; pp. 1362-1366.

17. Ricci, E.; Roberto, C.; Ippolito, A.; Bianco, A.; Scalise, M.T. A new pressure-relieving mattress overlay. EWMA J. $2013,13,1$.

18. Defloor, T.; De Bacquer, D.; Grypdonck, M.H. The effect of various combinations of turning and pressure reducing devices on the incidence of pressure ulcers. Int. J. Nurs. Stud. 2005, 42, 37-46. [CrossRef] [PubMed]

19. Sikka, M.P.; Garg, S. Functional textiles for prevention of pressure ulcers-A review. Res. J. Text. Appar. 2020, 24, 185-198. [CrossRef]

20. Mansfield, S.; Obraczka, K.; Roy, S. Pressure Injury Prevention: A Survey. IEEE Rev. Biomed. Eng. 2019, 13, 352-368. [CrossRef] [PubMed]

21. Tannen, A.; Dassen, T.; Halfens, R. Differences in prevalence of pressure ulcers between the Netherlands and GermanyAssociations between risk, prevention and occurrence of pressure ulcers in hospitals and nursing homes. J. Clin. Nurs. 2008, 17, 9. [CrossRef] [PubMed]

22. McInnes, E.; National Institite for Clinical Excellence. The use of pressure-relieving devices (beds, mattresses and overlays) for the prevention of pressure ulcers in primary and secondary care. J. Tissue Viability 2004, 14, 4-6. [PubMed]

23. Pan, P.; Haesler, E. Prevention and Treatment of Pressure Ulcers: Clinical Practice Guideline; Cambridge Media: Hong Kong, China, 2014.

24. Padula, W.V.; Mishra, M.K.; Makic, M.B.F.; Sullivan, P.W. Improving the Quality of Pressure Ulcer Care with Prevention: A Cost-Effectiveness Analysis. Med. Care 2011, 49, 385. [CrossRef] [PubMed]

25. Simon, J.P.; Patricia, W.S. A systematic review of economic evaluations assessing interventions aimed at preventing or treating pressure ulcers. Int. J. Nurs. Stud. 2015, 52, 769-788. [CrossRef] [PubMed] 
26. Schulz, S.; Pylatiuk, C.; Bretthauer, G. A new ultralight anthropomorphic hand. In Proceedings of the 2001 ICRA. IEEE International Conference on Robotics and Automation (Cat. No. 01CH37164), Seoul, Korea, 21-26 May 2001; pp. $2437-2441$.

27. Spetz, J.; Brown, D.S.; Aydin, C.; Donaldson, N. The value of reducing hospital-acquired pressure ulcer prevalence: An illustrative analysis. J. Nurs. Adm. 2013, 43, 235. [CrossRef] [PubMed]

28. Vanderwee, K.; Defloor, T.; Beeckman, D.; Demarré, L.; Verhaeghe, S.; Van Durme, T.; Gobert, M. Assessing the adequacy of pressure ulcer prevention in hospitals: A nationwide prevalence survey. BMJ Qual. Saf. 2011, 20, 260-267. [CrossRef] [PubMed]

29. Baumgarten, M.; Margolis, D.; Orwig, D.; Hawkes, W.; Rich, S.; Langenberg, P.; Shardell, M.; Palmer, M.H.; McArdle, P.; Sterling, R.; et al. Use of Pressure-Redistributing Support Surfaces Among Elderly Hip Fracture Patients Across the Continuum of Care: Adherence to Pressure Ulcer Prevention Guidelines. Gerontologist 2010, 50, 253-262. [CrossRef] 